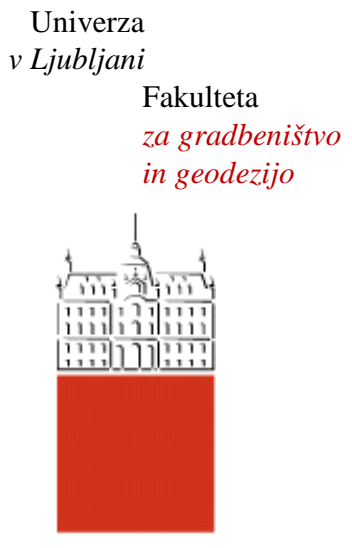

Jamova 2

1000 Ljubljana, Slovenija http://www3.fgg.uni-lj.si/

DRUGG - Digitalni repozitorij UL FGG http://drugg.fgg.uni-lj.si/

Ta članek je avtorjeva zadnja recenzirana različica, kot je bila sprejeta po opravljeni recenziji.

Prosimo, da se pri navajanju sklicujete na bibliografske podatke, kot je navedeno:

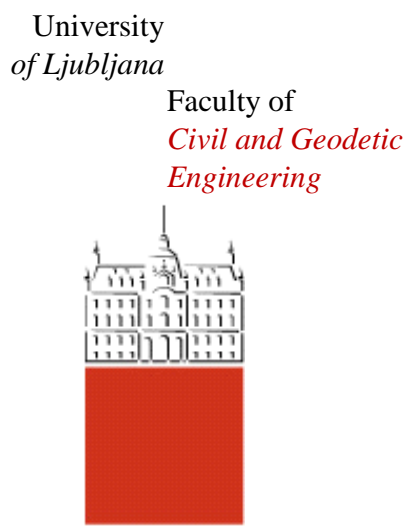

Jamova 2

SI - 1000 Ljubljana, Slovenia http://www3.fgg.uni-lj.si/en/

DRUGG - The Digital Repository http://drugg.fgg.uni-lj.si/

This version of the article is author's manuscript as accepted for publishing after the review process.

When citing, please refer to the publisher's bibliographic information as follows:

Ambrožič, T. in Turk, G. 2003. Prediction of subsidence due to underground mining by artificial neural networks. Computers \& Geosciences 29, 5: 627-637.

DOI: 10.1016/S0098-3004(03)00044-X. 


\title{
Prediction of subsidence due to underground mining by artificial neural networks
}

\author{
Tomaž Ambrožič, Goran Turk ${ }^{*}$ \\ University of Ljubljana, Faculty of Civil and Geodetic Engineering, \\ Jamova 2, 1000 Ljubljana, Slovenia
}

\begin{abstract}
Alternatively to empirical prediction methods, methods based on influential functions and methods based on mechanical model, artificial neural networks can be used for the the surface subsidence prediction. In our case, the multi-layer feed-forward neural network was used. The training and testing of neural network is based on available data. Input variables represent extraction parameters and coordinates of the points of interest, while the output variable represents surface subsidence data. After the neural network has been successfully trained, its performance is tested on a separate testing set. Finally, the surface subsidence trough above the projected excavation is predicted by the trained neural network. The applicability of artificial neural network for the prediction of surface subsidence was verified in different subsidence models and proved on actual excavated levels and in levelled data on surface profile points in the Velenje Coal Mine.
\end{abstract}

Keywords: Subsidence prediction; Artificial neural network; Multi-layer feed-forward neural network; Approximation of functions; Mining damage

\footnotetext{
* Corresponding author. Tel.: +386-1-4768-614; fax.: +386-1-4768-629.

E-mail address: gturk@fgg.uni-lj.si (G. Turk).
} 


\section{Introduction}

Underground mining causes the formation of surface subsidence trough. The prediction of the consequences of mining is an important task for the mine surveying service. The knowledge about the surface activity caused by mining, and the prediction of subsidence enable efficient repairs of the mining damage, and has a positive impact on the economic results of mining. Accurate and reliable prediction can, beside other factors, influence significantly the strategy of the operation of a mine. Due to a large number of parameters influencing the behaviour of the rock above the excavated space, the prediction of mining consequences is a demanding task. It is difficult to determine all the parameters, and it is even more difficult to determine their relative impact.

Displacements cause damage in different objects on the surface. Therefore the aim of mine surveyors at the beginning of the last century was to estimate the impact of underground mining on buildings, transport systems and surface above mines. They started to measure the displacements of points in the mine and on the surface, in order to be able to control the subsidence process and to diminish the damages caused by the excavation. They prescribed procedures of monitoring displacements and developed the methods for the prediction of surface subsidence in individual mines (Kratzsch, 1983). Several prediction methods have been developed.

The first methods for the prediction of surface subsidence were empirical prediction methods. These methods are based on the correlation of the measuring data and the related measuring results with the geometric parameters of the excavations (height and depth of the excavation or thickness and depth of the layer, as well as the quantity of the excavation, the location of the excavated edge, etc.). As these methods are derived 
from the measurements in a specific area, they are in direct relation to it, and the results are valid only for the investigated area. Examples of such methods are: the method of the angle of intersection, the Russian, Polish, Hungarian and the programmed profile curve methods, methods of the integration grid as well as the prediction model of the Velenje Coal Mine.

Prediction methods based on influential functions form the second group of prediction methods. The influential function is used to describe the value of the impact of elementary part of the excavation on the formation of subsidence. This group of prediction methods is based on seven assumptions or principles which simplify the calculus and make the methods generally applicable. The principle of using the methods is to select the influential function for each mine and then determine the coefficients in order to ensure that the subsidence curve is similar to the form of the subsidence in nature. The methods are simple and efficient, but it is difficult to calculate the coefficients in the equations of influential functions, as in nature the subsidence is influenced by numerous geomechanical and geological phenomena.

The third group of prediction methods consists of the model prediction methods. Their origin is in mathematical-physical models. The behaviour of roof and the development of subsidence are calculated according to the laws of mechanics. The elastic and plastic models of subsidence belong to this group of prediction methods. When using these models, the problem is usually solved by numerical methods, such as the finite element method, the finite difference method or the boundary element method.

An alternative to the above stated methods is the prediction of surface subsidence with artificial neural networks. An important advantage of such prediction of subsi- 
dence is that one does not need to know the geological and geomechanical conditions in the slope above the excavation. However, we need to have access to the data on excavations and the data on the displacements of points on the surface or the data on the causes of subsidising and the data on the consequences of excavations. It is much easier to get these data than to acquire all the influences needed for the previously mentioned methods of subsidence prediction.

\section{Artificial neural networks}

Artificial neural networks are networks, consisting of an arbitrary number of very simple elements, called neurons. Neurons, presented in the network as variables with the values of the momentary signals, are connected by connections. These are defined by weights. Through connections a neuron receives a signal from other neurons. The activation function intensifies or weakens the signal which is transmitted to other neurons.

Considering the geometry of the network, there are several types of neural networks: Hopfield, Hamming, Campenter and Grossberg, Kohonen, multi-layer feed-forward neural network and others (Lippmann, 1987; [1]). Neural networks with different geometry are used for solving various problems. The first three types of neural networks are usually used for binary input data and with problems of classification into classes. The last two types of neural networks are appropriate for the approximation of an unknown function. For the prediction of subsidence, the multi-layer feed-forward neural network is used, as our aim is to approximate an unknown relationship between the input and output data. The input data include the data on excavations and location coordinates of grid or profile points on the surface, and the output data are their height 
coordinates.

\subsection{Multi-layer feed-forward neural network}

The geometry of a multi-layer feed-forward neural network is shown in Fig. 1. Input units are connected to the first layer of hidden units which are further connected to the units of the second hidden layer. The units of the last hidden layer are connected to the output units. The multi-layer feed-forward networks are usually employed as the approximators of the unknown functional relation. In fact, it was shown in (Hornik et al., 1989) and (Funahashi, 1989) that any continuous function may be accurately approximated by the multi-layer feed-forward neural network.

The input units represent the input data, and the output units represent the output data. The hidden layers may be considered as a black box which performs the necessary transformations of the input data so that the target output data are obtained.

Each unit is represented by its value $y_{i}^{k}$. Each connection between the units is represented by its weight $w_{i j}^{k}$, where index $i$ corresponds to the unit number of the $k^{\text {th }}$ layer, while index $j$ corresponds to the unit number of the $(k-1)^{\text {th }}$ layer. The input layer is denoted by 0 , whereas the output layer is denoted by $n_{l}$. The signals travel in one direction only, i.e. from the input layer toward the output layer. The value of a unit is multiplied by the corresponding weight and added to the value of the signal in the unit of the next layer. In addition, the value of bias neuron or threshold $\vartheta_{i}^{k}$ is added to the equation

$$
y_{i}^{k}=\mathrm{f}\left(\sum_{j=1}^{n_{k-1}} w_{i j}^{k} y_{j}^{k-1}+\vartheta_{i}^{k}\right) .
$$

This equation is illustrated in Fig. 1. Activation function $\mathrm{f}($.$) enables the modelling of$ 
an arbitrary non-linear relation between input and output variables. Different functions could be used as an activation function. The usual choices of activation function are a sigmoid function

$$
\mathrm{f}(y)=\frac{1}{1+e^{-y}}
$$

$\tanh y$, or Gaussian. The behaviour of the neural network depends on the values of weights $w_{i j}^{k}$ and thresholds $\vartheta_{i}^{k}$ which have to be determined by the training (learning) procedure.

The set of known input and output values is termed an input-output pair. All inputoutput pairs are usually divided into three sets. The first is learning or training set which is used to determine the connection weights $w_{i j}^{k}$ and thresholds $\vartheta_{i}^{k}$. When the training procedure ends, after the neural network performs adequately for all inputoutput pairs in the training set, the neural network is assessed using the validation set of input-output pairs and the optimal neural network is chosen. Finally the chosen and taught neural network is tested, using the testing data set.

For numerical reasons the values of input and output units have to be normalized. The normalization of the values of output units depends on the range of activation function. Usually, the linear transformation works well, although sometimes a nonlinear transformation may help if the data are clustered.

The supervised training is in fact a general optimization problem in which the minimum of error $E_{p}$ is sought

$$
E_{p}=\frac{1}{2} \sum_{i=1}^{n_{o}}\left(t_{p i}-y_{p i}^{n_{l}}\right)^{2}
$$

where $t_{p i}$ are the target output values, $y_{p i}^{n_{l}}$ are the values of neurons in the output layer, i.e. the output values evaluated by neural network, $n_{o}$ is the number of neurons in 
output layer, i.e. the number of output variables.

Numerically this is a very demanding problem since there normally exists a large number of local minima. There are two essentially different approaches: error backpropagation algorithms which is basically a gradient method, and genetic algorithms which is in fact a stochastic search (Goldberg, 1989). There are many variations and combinations of the above mentioned method, see e.g. Treadgold and Gedeon (1998). If the number of weights is relatively small, the gradient method is a good choice. The error back-propagation or generalized delta rule as it was termed by its authors Rumelhart and McClelland (1986) is a gradient method in which the weights are changed for a chosen step size in the direction of the maximum descent for each input-output pair. However, there is always a possibility of finding only a local minimum which may not give satisfactory set of weights. One solution to this problem is simply to run the error back-propagation procedure for different starting points and then choose the best result.

In the error back-propagation the weights are changed in the direction of maximum descent of each input-output pair $p$

$$
\Delta w_{i j}^{k}=-\Delta_{w} \frac{\partial E_{p}}{\partial w_{i j}^{k}}, \text { and } \Delta \vartheta_{i}^{k}=-\Delta_{\vartheta} \frac{\partial E_{p}}{\partial \vartheta_{i}^{k}}
$$

where $\Delta_{w}$ is the step size defining the rate of changing the weights $w_{i j}^{k}$, and $\Delta_{\vartheta}$ is the step size defining the rate of changing the thresholds $\vartheta_{i}^{k}$. The derivatives in Eq. (4) are determined consecutively from the weights between the output layer and the last hidden layer towards the weight between the input layer and the first hidden layer by the chain rule

$$
\frac{\partial E_{p}}{\partial w_{i j}^{k}}=\frac{\partial E_{p}}{\partial y_{p i}^{k}} \frac{\partial y_{p i}^{k}}{\partial w_{i j}^{k}} \text { and } \frac{\partial E_{p}}{\partial \vartheta_{i}^{k}}=\frac{\partial E_{p}}{\partial y_{p i}^{k}} \frac{\partial y_{p i}^{k}}{\partial \vartheta_{i}^{k}}
$$


The derivatives $\partial y_{p i}^{k} / \partial w_{i j}^{k}$ and $\partial y_{p i}^{k} / \partial \vartheta_{i}^{k}$ are obtained from Eq. (1)

$$
\frac{\partial y_{p i}^{k}}{\partial w_{i j}^{k}}=\frac{d f\left(y_{p i}^{\prime k}\right)}{d y_{p i}^{\prime k}} y_{p j}^{k-1} \text { and } \frac{\partial y_{p i}^{k}}{\partial \vartheta_{i}^{k}}=\frac{d f\left(y_{p i}^{\prime k}\right)}{d y_{p i}^{\prime k}}
$$

In the case of neurons in the output layer the derivatives $\partial E_{p} / \partial y_{p i}^{k}$ are determined by the following equation which stems from (3)

$$
\frac{\partial E_{p}}{\partial y_{p i}^{n_{l}}}=-\left(t_{p i}-y_{p i}^{n_{l}}\right)
$$

In the case of all other neurons the derivatives are obtained from Eq. (1)

$$
\frac{\partial E_{p}}{\partial y_{p i}^{k-1}}=\sum_{j=1}^{n_{k}} \frac{\partial E_{p}}{\partial y_{p j}^{k}} \frac{\partial y_{p j}^{k}}{\partial y_{p i}^{k-1}}
$$

The process is repeated for each input-output pair $p$ until the error is smaller than prescribed for all input-output pairs. If the prescribed error is too small, overfitting may occur. Overfitting means that the neural network may reproduce input-output pairs used in the training procedure, but it fails to generalize them and may produce erroneous results, if some values of the input units are changed.

There are two major difficulties when using error back-propagation: it is almost impossible to choose the optimal step size, and quite often the procedure converges to a local minimum. If the step size is too large, we may overshoot the minimum. On the other hand, if the step size is too small, the convergence is very slow. Both difficulties may be overcome by different procedures with adaptive step size (Janakiraman and Honavar, 1993), or with the introduction of the inertial term (Lippmann, 1987).

The parameters, i.e. the number of hidden layers and the number of hidden neurons, of the optimal neural network are problem dependent. One of the methods how to choose the right network is by using the validation set to determine which one performs best. However, some general guidelines can be given. If the number of units is very 
large, the training procedure may be very slow, since each forward calculation takes a substantial computational effort. Although larger networks are usually able to learn the sought relationship, this may sometimes be a drawback. A large network may easily reproduce the training set of input-output pairs but it fails to generalize, yielding to a poor testing performance. Networks with insufficient units may have problems to learn properly during the training procedure.

\subsection{Use of neural network in mining}

The use of neural networks in engineering has become extremely widespread in the last few years. Let us describe only a few examples of using neural networks in mining and in similar expert fields.

Neural networks were used to determine ore boundary delineation, aggregate quality and rock indentation depth (Huang and Wänstedt, 1997; Huang and Wänstedt, 1998), ore reserve estimation (Wu and Zhou, 1993), and real-time roof pressure (Feng et al., 1996).

In geotechnical researches neural networks were also used for the approximation of oedometer curves (Turk et al., 2001) and as a constitutive model of several soils (Logar and Turk, 1997) and other materials (Ghaboussi et al., 1991).

In the field of satellite remote sensing, neural networks were used for the determination of different lithological regions (Hafner and Komac, 1998). There are some other works dealing with ore and rock characterisation (Cutmore et al., 1997; Millar and Hudson, 1994; Utt, 1999). Neural network can also serve as a tool which helps to determine the relative importance of the factors influencing the stability of underground 
objects according to their importance (Yang and Zhang, 1997). An interesting use of neural network is reported in Dysart and Pulli (1990), Finnie (1999) and Musil and Plesinger (1996) where neural networks were used to determine the event type (earthquake, quarry and mining blasts, chemical explosions, etc.) from the seismological data. Similar topic is covered also by in Rudajev and Č́́ž (1999) in which the mining tremor occurrence is estimated by ANN.

\section{Numerical examples}

The results of subsidence prediction using artificial neural network (ANN) are compared to the results of the stochastic method of subsidence (Todorović, 1986) and uniform prediction model of the Velenje Coal Mine (Medved, 1994). Finally, the efficiency of the ANN is checked also against actual results of subsidence measurements, caused by the underground mining in the Velenje Coal Mine.

The neural network is trained with input-output pairs of a training data set by the generalised delta rule. The iterations are repeated as long as the relative error in all input-output pairs is larger than 5\%. The relative error is obtained as the difference between the actual value and the value obtained by ANN, divided by the size of the actual value of the subsidence.

The success of ANN training is evaluated on a testing data set with the difference $\delta$, between the actual value of subsidence, and the value, obtained by neural network. For the assessment the following statistics are used:

- $\operatorname{minimum}$ value of differences $\delta_{\text {min }}=\min _{i} \delta_{i}$, 
- $\operatorname{maximum}$ value of differences $\delta_{\max }=\max _{i} \delta_{i}$,

- mean value of differences $\bar{\delta}=\frac{\sum_{i} \delta_{i}}{N_{i-o}}$,

- standard deviation of differences $s=\sqrt{\frac{\sum_{i}\left(\delta_{i}-\bar{\delta}\right)^{2}}{N_{i-o}-1}}$,

- mean deviation of differences $d=\frac{\sum_{i}\left|\delta_{i}-\bar{\delta}\right|}{N_{i-o}}$,

where $N_{i-o}$ is the number of input-output pairs.

During a preliminary research the comparison between the programmed profile curve method and the ANN approximation is caried out. The preliminary research lets us conclude the following: first, the use of neural networks is appropriate for different lengths and depths of the excavations, furthermore it is more appropriate to use a coarse grid of input-output pairs, the networks can be trained even if there is only one hidden layer of neurons, and the results are poorer if there are three or more hidden layers of neurons. In such case the optimal geometry of ANN results to be the one with two hidden layers, each containing 40 neurons.

\subsection{Comparison with stochastic model of subsidence}

The slope subsidence above the excavated space is to be predicted by using the stochastic model of subsidence. With the coordinates of these points and the calculated appertaining subsidences the input-output pairs of the training and testing data sets are formed.

Beside coordinates $Y$ and $X$ of the surface point of subsidence the input data consist also of width, length, depth and the excavated height of the excavation. The output data are the calculated subsidence or coordinate $Z$ of surface point. To prepare the training 
data set, eight different sizes of excavations (Table 1) are used. They are located at three different depths $(300 \mathrm{~m}, 350 \mathrm{~m}$ and $400 \mathrm{~m})$ and have different excavated heights (4 m, $5 \mathrm{~m}$ and $6 \mathrm{~m}$ ). The subsidence is presented in equidistant square grid of $11 \times 11$ points. In the training data set there are $N_{i-o}=8 \cdot 3 \cdot 3 \cdot 11 \cdot 11=8712$ input-output pairs. When calculating subsidences, other parameters of the excavations are the same for all excavations (the angle of dip is $0^{\circ}$, the subsidence factor is 1 , the influence angle in the direction of dip is $70^{\circ}$, and in the direction of strike is $55^{\circ}$, relative limit subsidence for the edge of subsidence in floor, roof and in the direction of strike is $1 \%$ of the maximum subsidence).

Based on the previously obtained experience, the neural network with two hidden layers with forty neurons per layer is selected. Thus the geometry of ANN is $6-40-$ $40-1$. There are $6 \cdot 40+40 \cdot 40+40 \cdot 1=1880$ connections between neurons and the same number of weights that the neural network needs to define in the training stage. Also, $40+40+1=81$ values of threshold are to be determined.

The testing of the trained neural network is carried out for different examples of the excavation. Fig. 2 presents the results of the prediction of subsidence caused by a $350 \mathrm{~m}$ wide and $400 \mathrm{~m}$ long excavation with the excavating height of $4.0 \mathrm{~m}$ and mean depth of $325 \mathrm{~m}$. In this case the testing statistics are as follows: $\delta_{\min }=-0.283 \mathrm{~m}$, $\delta_{\text {max }}=0.255 \mathrm{~m}, \bar{\delta}=0.039 \mathrm{~m}, s=0.093 \mathrm{~m}, d=0.065 \mathrm{~m}$, and correlation coefficient $r=0.997$ (see Fig. 3).

In addition to the previously mentioned parameters the angle of dip was added as one of the parameters influencing the subsidence. All the treated excavations are calculated for three angles of dip: $0^{\circ}$ (already calculated), $10^{\circ}$ and $20^{\circ}$. If the subsidence 
was transposed into a grid of $11 \times 11$ points, there would be $N_{i-o}=8712 \cdot 3=26136$ input-output pairs in the training data set. Due to the large number of input-output pairs the decision was taken not to present the subsidence in a grid, but by point subsidences of the selected profile. The selected profile is presented in Fig. 4. The selected (main) profile consists of eleven grid points: from grid point No. 6 to grid point No. 116 . With the profile presentation of the subsidence there are $N_{i-o}=11 \cdot 8 \cdot 3 \cdot 3 \cdot 3=2376$ input-output pairs in the training data set.

Since various values of the angle of dip of the levels (inclined excavations) are given, a neuron representing the angle of dip is added into the input layer. Thus there are seven neurons in the input layer. The geometry of ANN is $7-40-40-1$.

The testing of the trained neural network is performed on several profiles. Fig. 5 shows the test results of the excavation with the width of $200 \mathrm{~m}$, length of 450 $\mathrm{m}$, excavation height of $5.5 \mathrm{~m}$, excavation depth of $325 \mathrm{~m}$ and angle of dip $0^{\circ}\left(1^{\text {st }}\right.$ case), and the test results of the excavation with the width of $450 \mathrm{~m}$, length of $450 \mathrm{~m}$, excavation height of $5.5 \mathrm{~m}$, excavation depth of $375 \mathrm{~m}$ and angle of dip $15^{\circ}$ ( $2^{\text {nd }}$ case). The statistics of this testing are presented in Table 2.

\subsection{Comparison with uniform prediction model of the Velenje Coal Mine}

The prediction of slope subsidence above the excavated levels with neural networks is continued with a subsidence obtained by uniform prediction model of the Velenje Coal Mine.

For the training of ANN, 33 levels of the southern wing of the cave Preloge in the 
Velenje Coal Mine were used with the following parameters (Fig. 6):

- level heights or names from ET.k.+100 up to ET.k.-65/D,

- level widths from $62 \mathrm{~m}$ up to $141 \mathrm{~m}$,

- level lengths from $82 \mathrm{~m}$ up to $853 \mathrm{~m}$,

- middle level depths from $260 \mathrm{~m}$ up to $425 \mathrm{~m}$,

- angle of dip - inclination of all levels is equal to $0^{\circ}$,

- excavation heights of levels from $8.0 \mathrm{~m}$ up to $13.9 \mathrm{~m}$,

- subsidence factor 0.86 ,

- average residual angle of internal friction $23^{\circ}$.

In Velenje Coal Mine the levels are labeled by "ET.k.+ $h$ ", where $h$ is the elevation above the see level in meters. The same labels of levels are kept in this paper.

The influential area due to the excavation of all levels is covered by a network of grid points. In these points the subsidences that occur due to the excavation in an individual level are calculated. Beside the coordinates of grid points the input data consist also of level length and width, rotation of the main axis of the level in the coordinate system, excavation height of the level, multiplied by the subsidence factor, coordinates of levels $Y$ and $X$ centroide, and level depth. The geometry of ANN is $9-40-40-1$. Thus, there are nine neurons in the input layer, two hidden layers with forty neurons in each hidden layer and one neuron in the output layer.

When the subsidence is treated by grids, the influential area is covered by a grid consisting of $20 \times 20$ grid points. Since there are 33 levels used for the training, the 
selected grid would yield 13200 input-output pairs in the training data set. Due to the large number of input-output pairs we decided to decrease their number by removing them randomly. Thus there remain 7989 or $60 \%$ of the input-output pairs in the training data set.

For the testing of neural network we used the level ET.k.-80/A of the southern wing of the cave Preloge with the following parameters (in Fig. 6 the test level is marked):

- level width $101 \mathrm{~m}$,

- level length $794 \mathrm{~m}$,

- middle level depth $440 \mathrm{~m}$,

- angle of dip - level inclination $0^{\circ}$,

- excavation height of the level $11.0 \mathrm{~m}$,

- subsidence factor 0.86 ,

- average residual angle of internal friction $23^{\circ}$.

The test results are presented in Fig. 7. The figure shows that the predicted subsidence is to a large extent similar to the one calculated by the neural network. In this case the testing statistics are as follows: $\delta_{\min }=-0.300 \mathrm{~m}, \delta_{\max }=0.180 \mathrm{~m}$, $\bar{\delta}=-0.004 \mathrm{~m}, s=0.071 \mathrm{~m}, d=0.044 \mathrm{~m}$, and correlation coefficient $r=0.998$ (see Fig. 8). 


\subsection{Prediction based on long term measurements}

Finally, the subsidence of the slope above the excavated levels is to be predicted using the data supplied by the mine surveying service of the Velenje Coal Mine. The data can be divided into two groups.

The first complex consists of the data pertaining to the points in the selected profiles, stabilised by iron wedges (Fig. 6), i.e. location coordinates of the point, levelling year and levelled altitude of the point. The data are available for 37 points located in four profiles. The second complex consists of the data pertaining to these levels (Fig. 6). These are: level name, indicating also its altitude, coordinates of corner and fraction points of the level corners, excavating height of the level and subsidence factor, excavating direction in the level and the date of the first and the last day of excavation in the level. We use data on 33 levels of the southern wing of the cave Preloge in Velenje Coal Mine that were being excavated between 1973 and 1997. The available data allow us to form input-output pairs of the training and testing data sets.

From the coordinates of corner and fraction points of level corners the rectangular levels are calculated in such way that the new rectangular levels, with the same surface as the real ones, match the real levels as much as possible. The transformation needs to be carried out due to different shapes of levels (from almost regular rectangular, presented with four points up to the shape given by twenty-two points). From the corner points the coordinates of transformed rectangular forms of levels, the lengths and widths of levels, rotations of main level axes in the coordinate system and coordinates of the levels $Y$ and $X$ centroides are calculated. The stated parameters of levels present the first five input variables necessary for the ANN training and testing procedure. The 
sixth input variable is the excavating height of each level multiplied by subsidence factor. For the subsidence factor the value 0.86 is assumed. The seventh input variable is the depth of the level, calculated from the altitude of each level. It is assumed that the surface is $360 \mathrm{~m}$ above the sea.

Based on the analysis of level excavation it is established that in almost all the years the subsidence of each surface point is influenced by two levels. This finding dictates the form of the data file with input-output pairs of training and testing data sets. They are composed in such a way that two levels influence the subsidence of each levelled point. Since individual level is described by seven parameters, each input-output pair consists of seventeen variables. The first sixteen variables pertain to input data. The first seven relate to the first influencing level, the second to the second level, and the fifteenth and the sixteenth input variables to coordinates $Y$ and $X$ of the chosen profile point on surface. The seventeenth variable represents the output data: the levelled subsidence of the chosen profile point on the surface in a certain year.

The geometry of ANN is chosen to be $16-40-40-1$, where the number of neurons in the input layer corresponds to the number of input variables, and one neuron in the output layer represents the output data. Each of the two hidden layers consists of forty neurons.

The ANN training and testing is carried out gradually. First the neural network is trained with the data of five years of excavations (from 1974 to 1978). Then the trained neural network is tested for the sixth year (1979). The obtained predicted subsidences of points are compared to the levelled results (obtained in 1979) and the successfulness of the training is assessed. Then the neural network is trained with the data obtained 
in the first six years of excavations (from 1974 to 1979) and the subsidences in the seventh year (1980) is predicted and tested with the measured values in 1980. The procedure is repeated for all periods for which the data on levels and levelled heights of points on the surface are available for. In the last example of training, the excavated levels are used from ET.k.+100 to ET.k.-65/D, and the prediction is tested for level ET.k.-80/A. The test results are presented in Fig. 9.

Since there were three or four levels influencing the point subsidence between 1981 and 1984, the data on the levels and levelling in this time frame are not considered in the process of the ANN training.

Fig. 9 and Fig. 10 show that the prediction of the subsidence of surface points using ANN is completely satisfactory, with the exception in the years 1979, 1986, 1988 and 1994, when some deviations of the values obtained by ANN from the levelled data can be noticed. The reasons for the deviations are as follows:

- in 1979 the surface points were influenced by the excavated level ET.k.+60 that was excavated in 1978, and it was located much more to the east than all levels excavated by then;

- in 1986 and 1988 the points on the surface were influenced by excavated levels in the eastern part of the southern wing of the cave Preloge; by then all the levels had been excavated in the western part;

- in 1994 level ET.k.-45/B caused chaos in the ANN training; it was excavated in 1993, the level centroide was located in the eastern most part of the treated levels.

All the calculated and four presented profile points let us conclude that the ANN training was successful, since the results of point subsidence are very similar to the 
levelled data. The largest deviation between the calculated results obtained by the trained neural network and the levelled data is in the point X1 in 1997 and it amounts to $34 \mathrm{~mm}$, which is less than $10 \%$ of the subsidence - the final subsidence at the point $\mathrm{X} 1$ is $383 \mathrm{~mm}$. The correlation coefficient is 0.9980 in the training phase and 0.9873 in the testing phase.

\section{Conclusion}

The paper deals with the prediction of surface subsidence due to underground mining using neural networks, which is a novelty, as up to the present time only empirical prediction methods, prediction methods based on influential function and model prediction methods have been used. The research used a multi-layer feed-forward neural network. The applicability of ANN for the prediction of surface subsidence was first checked in various models (model of subsidence according to programmed profile curve method, stochastic model and uniform prediction model of the Velenje Coal Mine). We concluded that ANN with two hidden layers with forty neurons in each layer gives satisfactory results. It was established that the successfulness of prediction does not depend on the manner of subsidence presentation. Small deviations between the predicted and the expected - measured values were obtained when the subsidences were presented in a grid of points or with points distributed in profiles. The practical applicability of the neural network was shown in real condition of excavated levels and levelled data on surface profile points in the Velenje Coal Mine. The result obtained by neural networks does not depend on geological and geomechanical conditions of roof. This, of course, is an advantage, as it is normally difficult to determine which parame- 
ters influence the subsidence, and it is even more difficult to determine the magnitude of their impact.

\section{Acknowledgement}

We would like to thank the team of the Mine Surveying Service in the Velenje Coal Mine for supplying the data on levels and profile points.

\section{References}

Cutmore, N.G., Liu, Y., Middleton, A.G., 1997. Ore characterisation and sorting. Minerals Engineering 10 (4), 421-426.

Dysart, P.S., Pulli, J.J., 1990. Regional seismic event classification at the noress array: Seismological measurements and the use of trained neural networks. Bulletin of the Seismological Society of America 80 (6), 1910-1933.

Feng, X.T., Wang, Y.J., Yao, J.G., 1996. A neural network model for real-time roof pressure prediction in coal mines. International Journal of Rock Mechanics and Mining Science \& Geomechanics Abstracts 33 (6), 647-653.

Finnie, G.J., 1999. Using neural networks to discriminate between genuine and spurious seismic events in mines. Pure and Applied Geophysics 154 (1), 41-56.

Funahashi, K.-I., 1989. On the approximate realization of continuous mappings by neural networks. Neural Networks 2, 183-192.

Ghaboussi, J., Garret, J.H.Jr., Wu, X., 1991. Knowledge-based modeling of material 
behavior with neural networks. ASCE, Journal of Engineering Mechanics 117 (1), $132-153$.

Goldberg, D.E., 1989. Genetic Algorithms in Search, Optimization, and Machine Learning, Addison-Wesley Publishing Company, Reading, 412pp.

Hafner, J., Komac, M., 1998. Landsat TM lithological classification of Koper - Kozina area. Neural network approach versus statistical clustering. In: Proceedings of International Conference on GIS for Earth Science Applications, Ljubljana, Slovenia, pp.41-55.

Hornik, K., Stinchcombe, M., White, H., 1989. Multilayer feedforward networks and universal approximations. Neural Networks 2, 359-366.

Huang, Y., Wänstedt, S., 1997. The use of artificial neural networks for the delineation of boundaries between ore bodies based on geophysical logging data. Mineral Resources Engineering 6 (1), 1-15.

Huang, Y., Wänstedt, S., 1998. The introduction of neural network system and its applications in rock engineering. Engineering Geology 49, 253-260.

Janakiraman, J., Honavar, V., 1993. Adaptive learning rate for increasing learning speed in backpropagation networks. In: Proceedings of World Congress on Neural Networks, Portland, Oregon, pp.IV-378-IV-381.

Kratzsch, H., 1983. Mining Subsidence Engineering, Springer-Verlag, Berlin, Heidelberg, New York, 543pp. 
Lippmann, R.P., 1987. An introduction to computing with neural nets. IEEE ASSP Magazine 4 (2), 4-22.

Logar, J., Turk, G., 1997. Neural network as a constitutive model of soil. Zeitschrift für Angewandte Mathematik und Mechanik 77, 195-196.

Medved, M., 1994. Prispevek k poznavanju degradacije okolja pri jamskem pridobivanju debelih slojev premoga (On environment degradation due to sublevel caving of thick lignite coal seam). Ph.D. Dissertation, University of Ljubljana, FNT, Ljubljana, $164 \mathrm{pp}$.

Millar, D.L., Hudson, J.A., 1994. Performance monitoring of rock engineering systems using neural networks. Transactions - Institution of Mining and Metallurgy. Section A. Mining industry 103, A3-A16.

Musil, M., Plesinger, A., 1996. Discrimination between local microearthquakes and quarry blasts by multi-layer perceptrons and Kohonen maps. Bulletin of the Seismological Society of America 86 (4), 1077-1090.

Rudajev, V., Číž, R., 1999. Estimation of mining tremor occurrence by using neural networks. Pure and Applied Geophysics 154 (1), 57-72.

Rumelhart, D.E., McClelland, J.L., 1986. Parallel Distributed Processing, Volume 1: Foundations, The MTI Press, Cambridge, 547pp.

Todorović, R., 1986. Prognoza parametrov deformacije površine nad rudarskimi pridobivalnimi deli v specifičnih pogojih slovenskih premogovnikov (The determination of 
parameters of surface subsidence above underground excavation in specific conditions of Slovenian coal mines). Ph.D. Dissertation, University of Ljubljana, FNT, Ljubljana, $131 \mathrm{pp}$.

Treadgold, N.K., Gedeon, T.D., 1998. Simulated annealing and weight decay in adaptive learning: The SARPROP algorithm. IEEE Transactions on Neural Networks 9 (4), 662-668.

Turk, G., Logar, J., Majes, B., 2001. Modelling soil behaviour in uniaxial strain conditins by neural networks. Advances in Engineering Software 32 (10-11), 57-64.

Utt, W.K., 1999. Neural network technology for strata strength characterization. In: Proceedings of International Joint Conference on Neural Netoworks, Washington D.C., paper 752 .

Wu, X., Zhou, Y., 1993. Reserve estimation using neural-network techniques. Computers \& Geosciences 19 (4), 567-575.

Yang, Y., Zhang, Q., 1997. A hierarchical analysis for rock engineering using artificial neural networks. Rock Mechanics and Rock Engineering 30 (4), 207-222.

\section{Internet references}

[1] Sarle, W.S. (Ed.), 2002. Neural network FAQ. ftp://ftp.sas.com/pub/neural/FAQ.html 


\section{List of figures}

Figure 1 Multi-layer feed-forward artificial neural network

Figure 2 Subsidence obtained by ANN and stochastic model

Figure 3 Correlation chart - ANN to stochastic model

Figure 4 The main profile for subsidence presentation and evaluation

Figure 5 Subsidence of the main profile obtained by ANN and stochastic model

Figure 6 Training and testing excavation, and profile points in the Coal Mine Velenje

Figure 7 Results of testing on level ET.k.-80/A

Figure 8 Correlation chart - ANN to prediction model of the Coal Mine Velenje

Figure 9 Subsidence of chosen profile points

Figure 10 Statistics of the differences between ANN prediction and actual subsidence 
$4^{\text {th }}$ neuron in the $1^{\text {st }}$ hidden layer
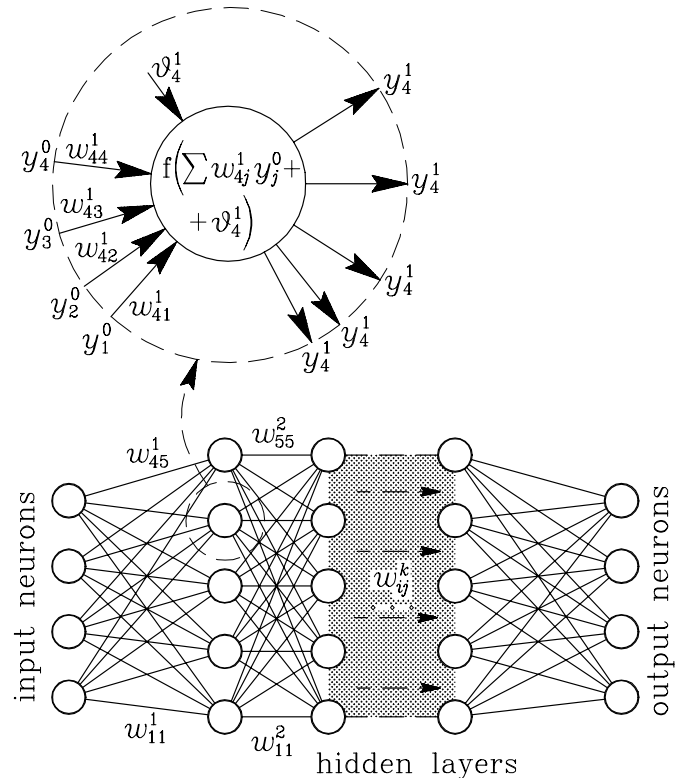

$\begin{array}{llcc}0 & 2 & n_{l}-1 & n_{l} \\ \text { layer } & \text { numbers }\end{array}$

Fig. 1 
Subsidences

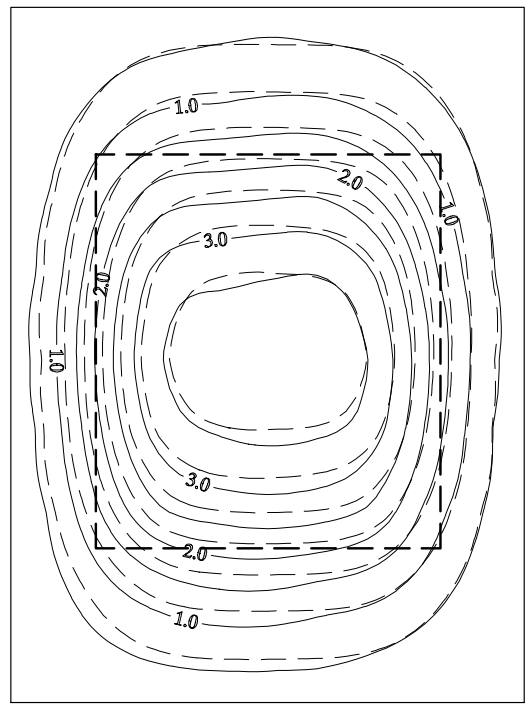

ANN prediction

- - - Stochastic model
Subsidence diference

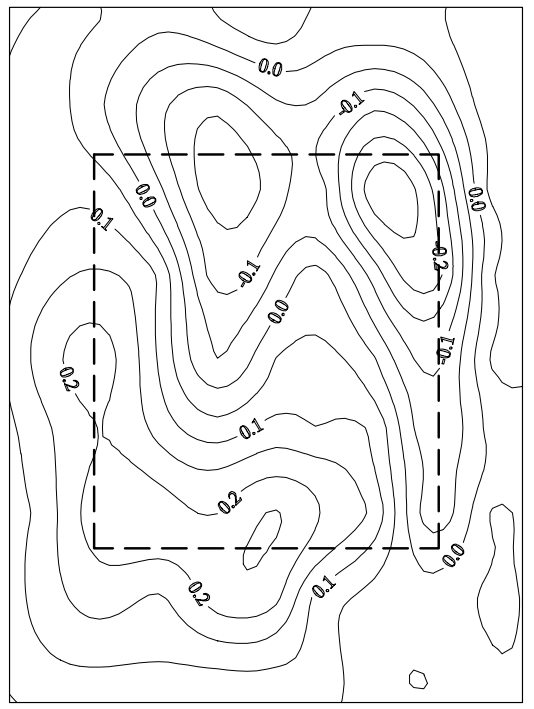

Fig. 2 


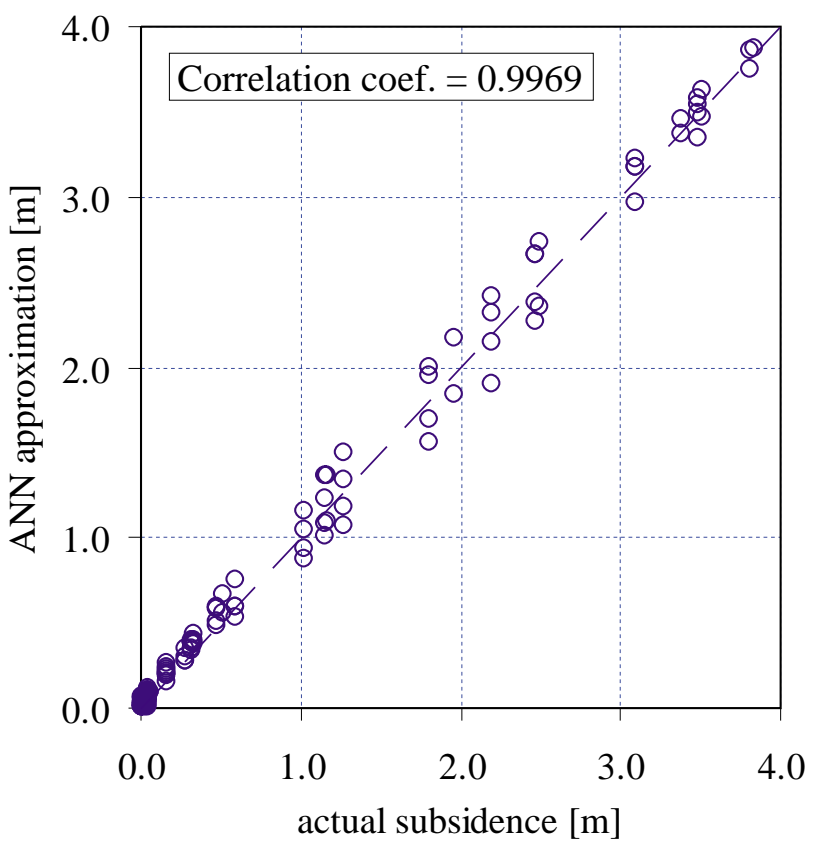

Fig. 3 


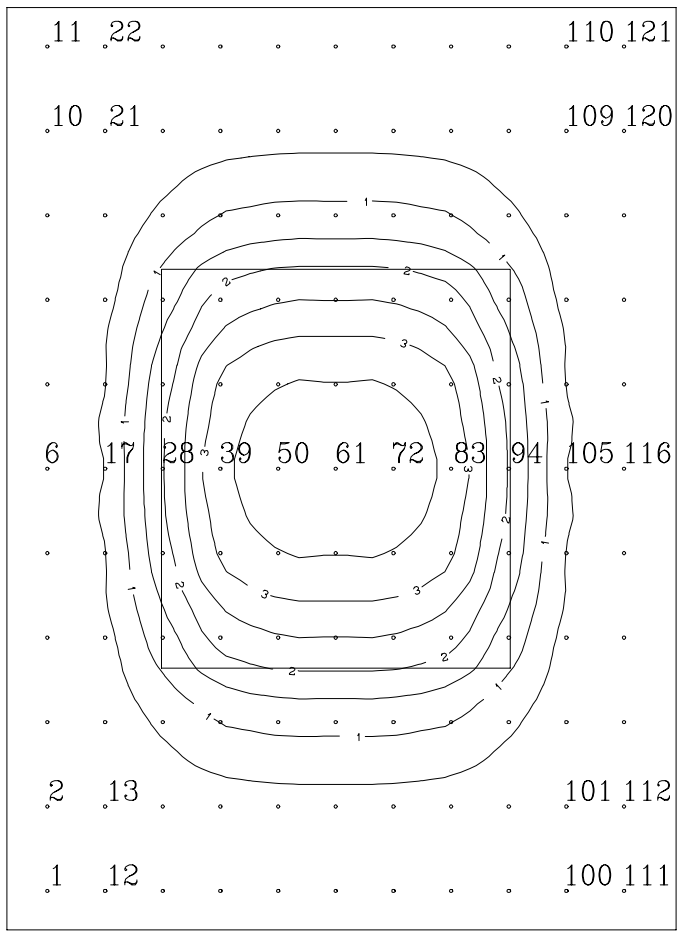

Fig. 4 
Excavation width $200 \mathrm{~m}$

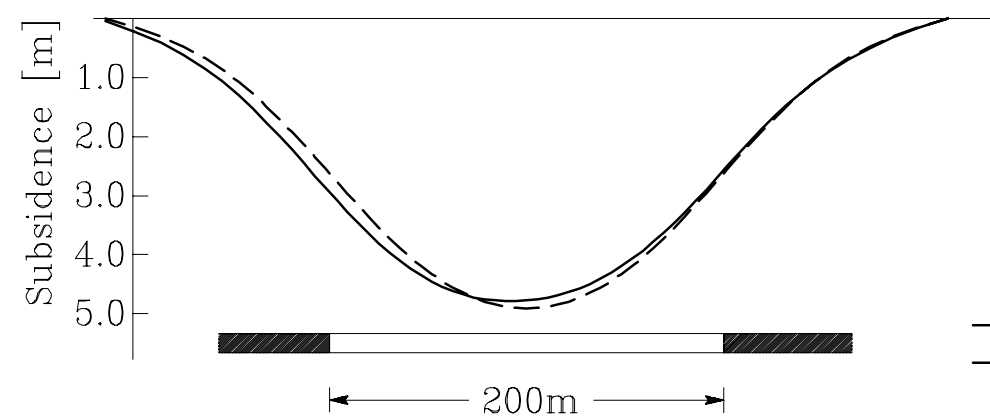

ANN prediction

- - Stochastic prediction

Excavation width $450 \mathrm{~m}$, excavation slope $15^{\circ}$

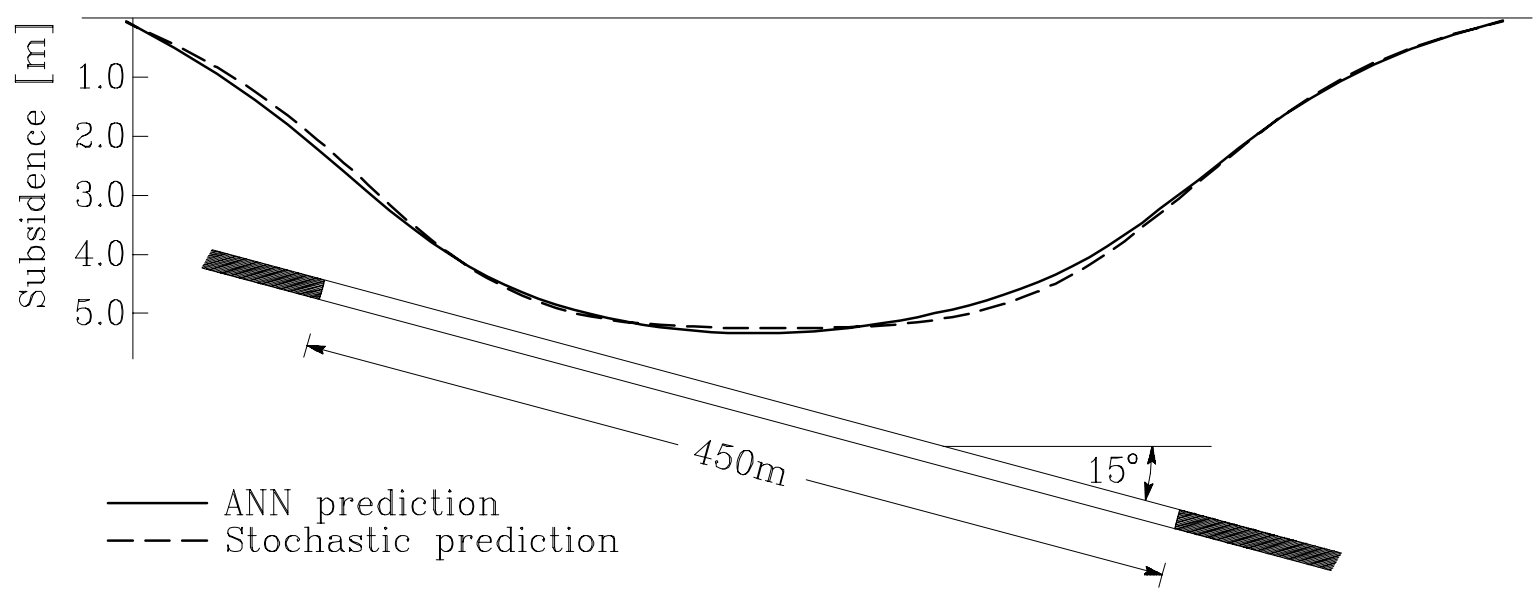

Fig. 5 


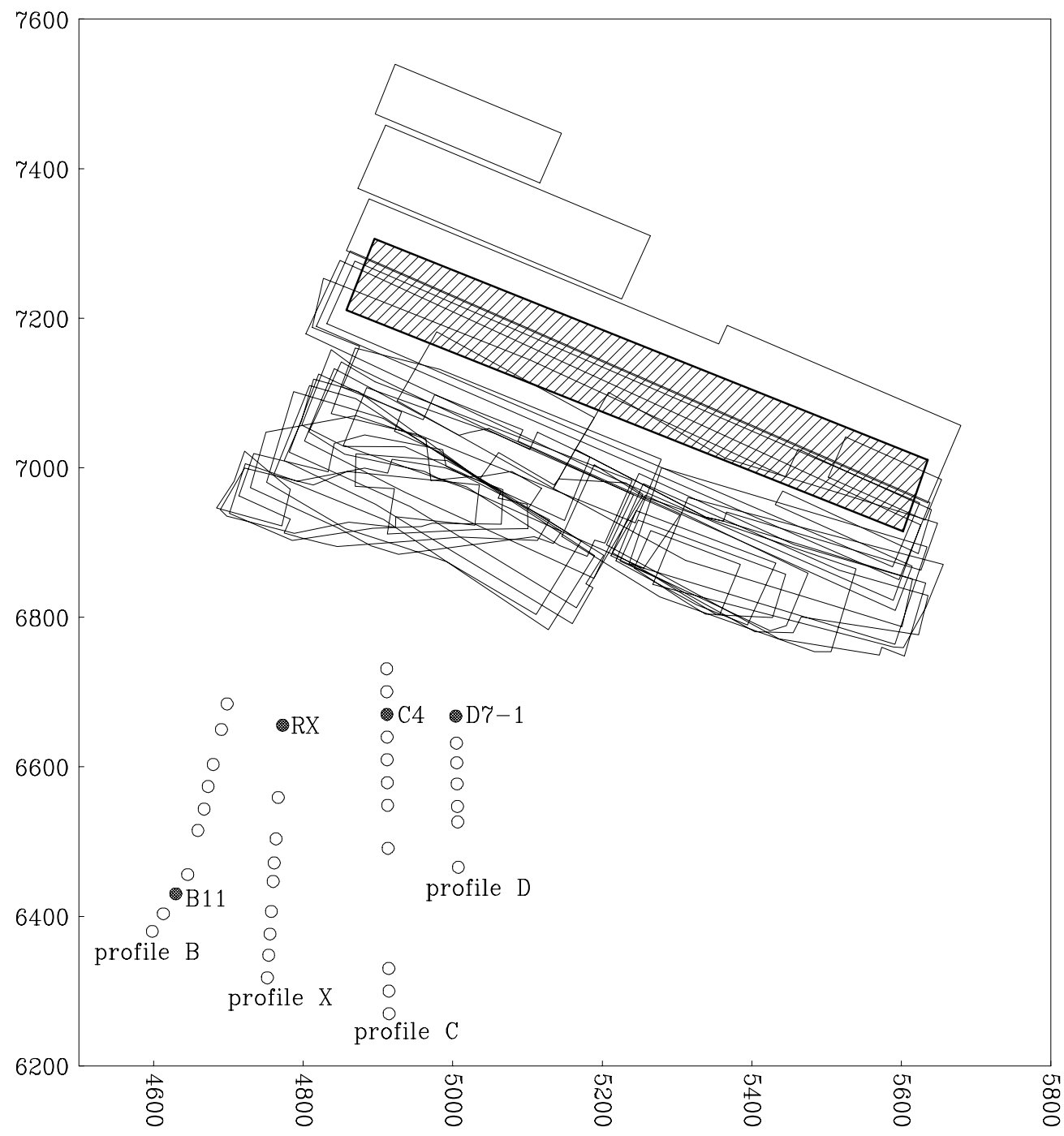

Fig. 6 


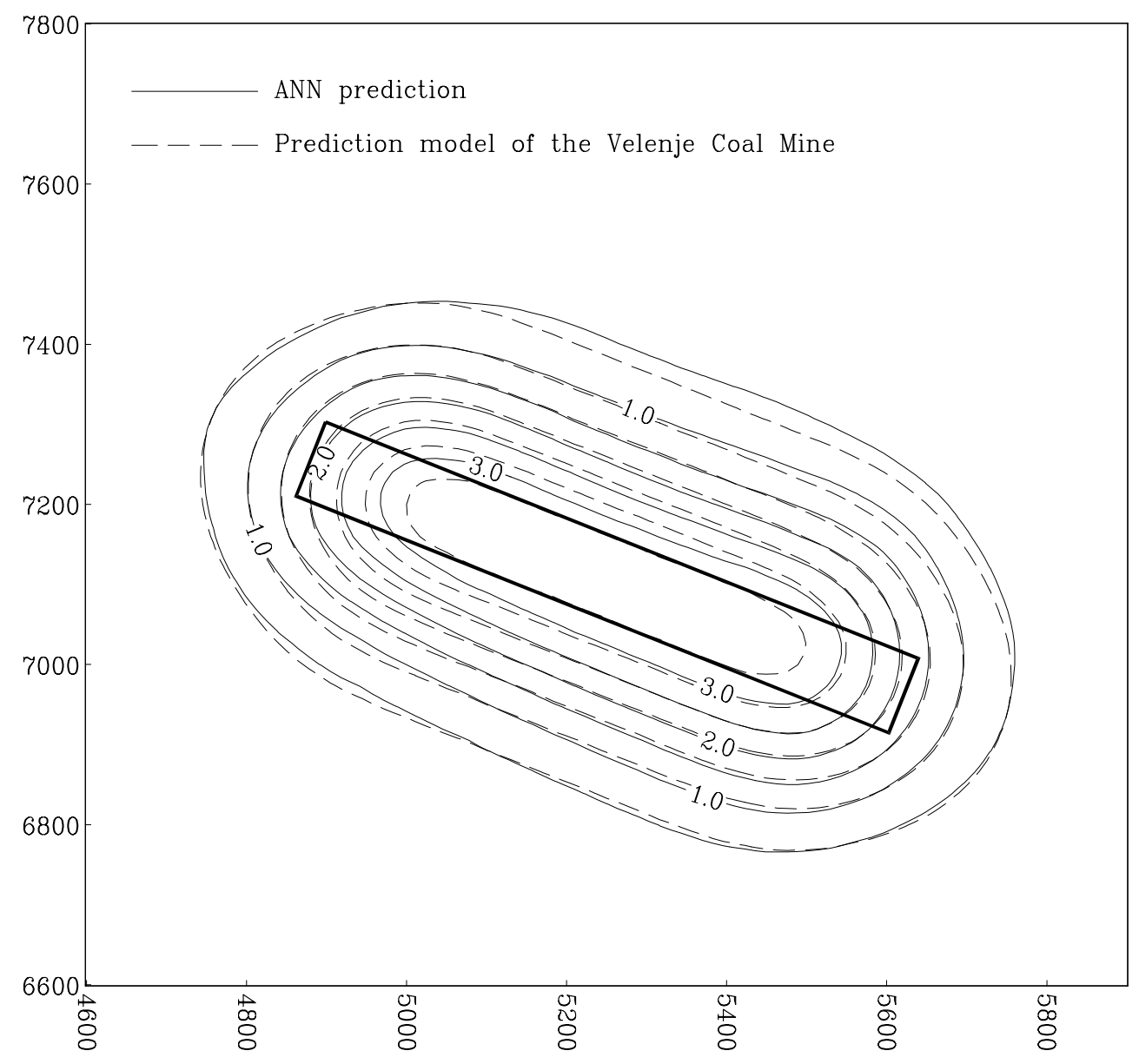

Fig. 7 


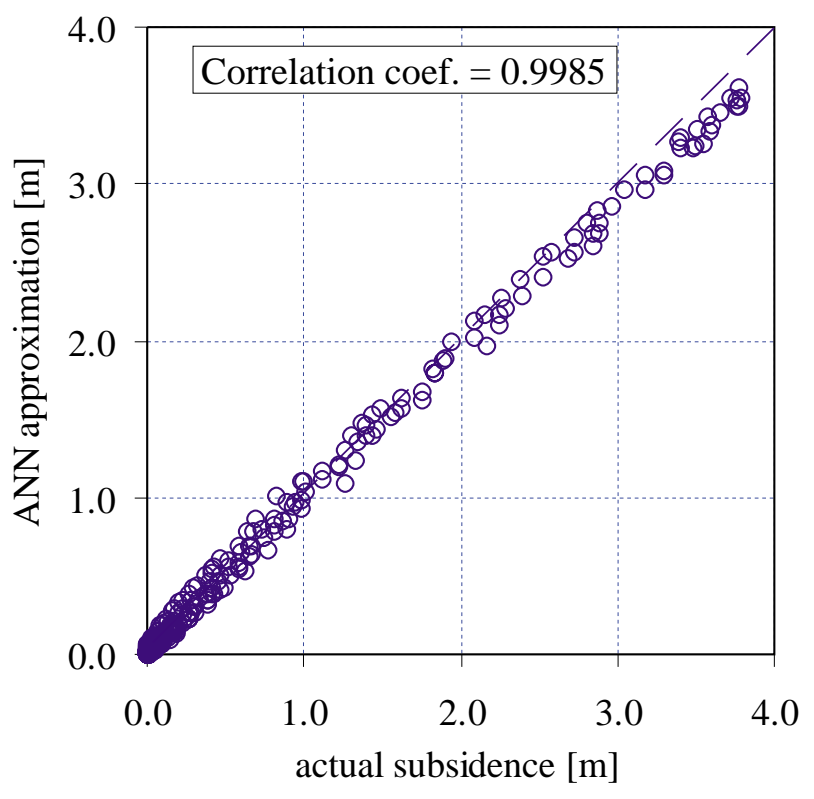

Fig. 8 


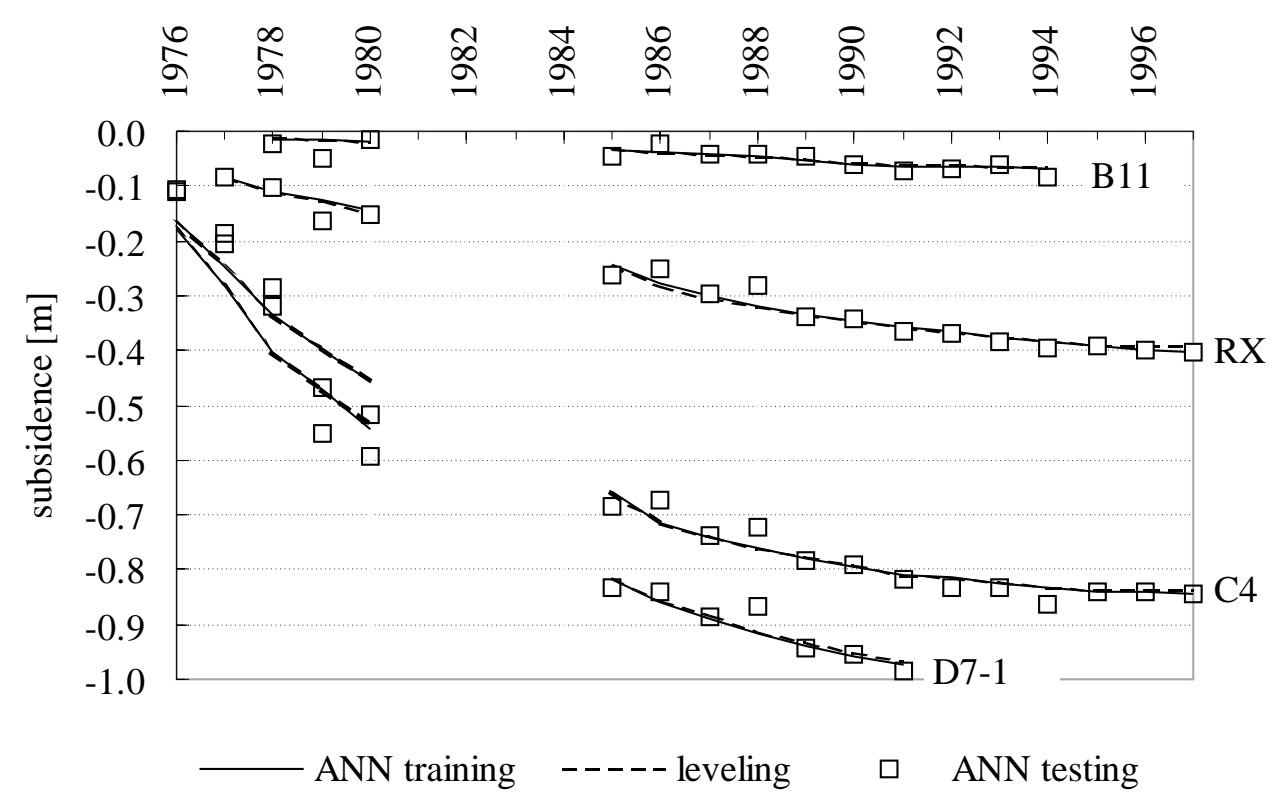

Fig. 9 


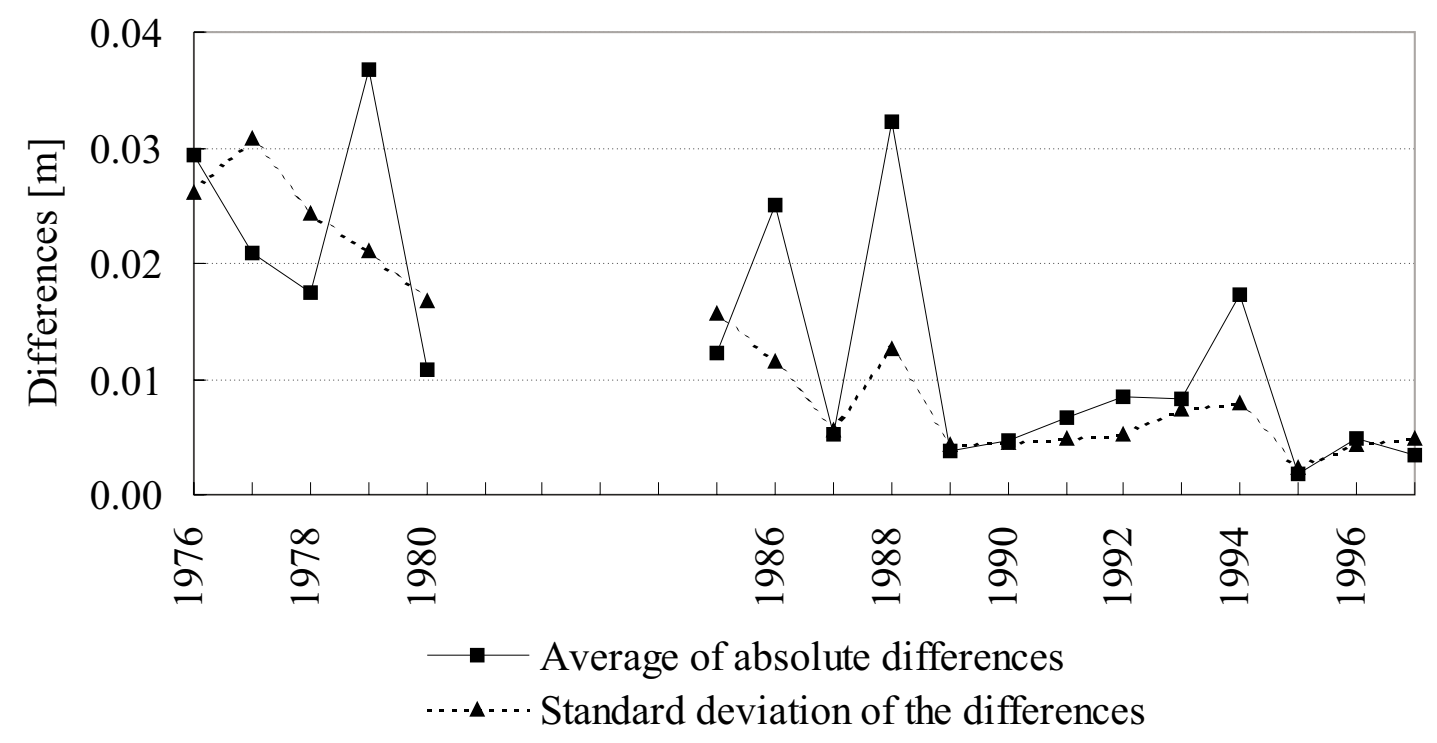

Fig. 10 


\section{List of tables}

Table 1 Value of widths $W$ and lengths $L$ of the excavations in the training procedure

Table 2 Statistics of the differences between ANN approximation and actual subsidences 
$W[\mathrm{~m}] \quad 200 \quad 200 \quad 250 \quad 300 \quad 300 \quad 350 \quad 400 \quad 400$

$L[\mathrm{~m}] \quad 400 \quad 500 \quad 450 \quad 400 \quad 500 \quad 450 \quad 400 \quad 500$ 


\begin{tabular}{cccccc}
\hline & $\delta_{\min }[\mathrm{m}]$ & $\delta_{\max }[\mathrm{m}]$ & $\bar{\delta}[\mathrm{m}]$ & $s[\mathrm{~m}]$ & $d[\mathrm{~m}]$ \\
\hline $1^{\text {st }}$ case & -0.181 & 0.349 & 0.049 & 0.160 & 0.126 \\
$2^{\text {nd }}$ case & -0.182 & 0.222 & 0.006 & 0.121 & 0.100 \\
\hline
\end{tabular}

